\title{
Association between single nucleotide variants of vascular endothelial growth factor $A$ and the risk of thyroid carcinoma and nodular goiter in a Han Chinese population
}

\author{
Rui Liu ${ }^{1, *}$, Lifeng Ning ${ }^{1,2, *}$, Xiaoli Liu ${ }^{3}$, Huiping Zhang ${ }^{4}$, Yaqin Yu ${ }^{1}$, Shangchao \\ Zhang ${ }^{1}$, Wenwang Rao ${ }^{1}$, Jieping Shi ${ }^{1}$, Hui Sun ${ }^{3}$, Qiong Yu ${ }^{1}$ \\ ${ }^{1}$ Department of Epidemiology and Biostatistics, School of Public Health, Jilin University, Changchun 130021, China \\ ${ }^{2}$ National Research Institute for Family Planning, Beijing 100081, China \\ ${ }^{3}$ Jilin Provincial Key Laboratory of Surgical Translational Medicine, China-Japan Union Hospital, Jilin University, Changchun \\ 130033, China \\ ${ }^{4}$ Department of Psychiatry, Yale University School of Medicine, New Haven, CT 06511, USA \\ *These authors have contributed equally to this work \\ Correspondence to: Qiong Yu, email: yuqiong@jlu.edu.cn
}

Keywords: papillary thyroid carcinoma, nodular goiter, VEGFA, SNPs, Han Chinese

Received: September 01,2016 Accepted: January 06, $2017 \quad$ Published: February 02, 2017

\section{ABSTRACT}

The aim of the present study was to investigate whether genetic variants in the vascular endothelial growth factor A gene (VEGFA) were risk factors for papillary thyroid carcinoma (PTC) or nodular goiter (NG) in Han Chinese. A total of 2,319 subjects ( 861 PTC patients, 562 NG patients, and 896 healthy controls) were included. Five tag single nucleotide polymorphisms (tagSNPs: rs3024997, rs3025040, rs833070, rs25648, and rs10434) in VEGFA were genotyped. SNP rs3025040 T allele was associated with a decreased risk of NG $(P<0.05)$. SNP rs3024997 was associated with an increased risk of PTC $(P<0.05)$ and NG $(P<0.001)$ when an over-dominant model (AA+GG vs. AG) was considered. PTC patients carry the less frequent TT genotype (compared to the CC genotype) $(P<0.05)$ of SNP rs3025040. Likewise, NG patients have the less frequent TC genotype compared to the CC $(P<0.05)$. No significant association of SNPs rs833070, rs25648, and rs10434 with PTC or NG was observed. Haplotypes AT (rs3024997 and rs3025040) and GTA (rs10434, rs3025040, and rs3024997) showed a lower risk for NG $(P<0.01$ and $P<0.05$, respectively), while haplotypes GTT (rs833070, rs3025040, and rs3024997) and GGGT (rs833070, rs10434, rs3024997, and rs3025040) predicted the risk of progression to NG (both $P$ <0.05). Haplotype AGAC (rs833070, rs10434, rs3024997, and rs3025040) conferred protection for PTC $(P<0.05)$. In summary, this study indicated for the first time that SNPs rs3024997 and rs3025040 in VEGFA were significantly associated with PTC and/ or NG. Haplotypes of the VEGFA may influence the risk of PTC and NG.

\section{INTRODUCTION}

Thyroid cancer is one of the most common endocrine malignancies with a fairly rapid rising incidence throughout the world in recent years [1]. Over the past three decades, the incidence of thyroid cancer has nearly tripled, and almost all of the increase in the incidence of thyroid cancer over the years was due to the increase of the prevalence of PTC [2]. Numerous studies have indicated that complicated interactions between environmental and genetic factors contribute to PTC
[3-5]. The risk factors for PTC include old age, extent of lymph node metastases, extra thyroidal invasion, distant metastasis, aggressive histological subtype, large tumor size, and terminal stage [6]. Although the prognosis of PTC appears to be relatively better than other types of cancer, cervical lymph node metastases and aggressive subset are the main contributors to poor prognosis [7]. NG is a common pathology of the thyroid gland, and varies in incidence in different parts of the world [8]. The incidence of thyroid cancer in multinodular goiter is estimated to be $5-10 \%$ [9]. The risk factors for NG include goiter size, 
Table 1: Baseline characteristics of subjects

\begin{tabular}{lccc}
\hline Group & n & Gender (Male/Female) & Age* \\
\hline PTC & 861 & $209 / 652$ & $44(38-49)$ \\
NG & 562 & $138 / 424$ & $49(42-56)$ \\
Controls & 896 & $220 / 676$ & $43(38-49)$ \\
\hline
\end{tabular}

*Age, median (interquartile range) years.

thyrotrophin, multifocal, heterogeneous, nodular growth pattern, and genetic predisposition [10]. A number of studies have investigated the association between PTC or NG and single nucleotide polymorphisms (SNPs) within or nearby candidate genes $[9,11]$. In recent years, many researchers have investigated the genetic susceptibility to PTC, and several susceptibility genes for PTC have been identified [12].

The vascular endothelial growth factor A (VEGFA or VEGF) is one of the most potent inducers of growth factors. VEGF family members include VEGFA, VEGFB, VEGFC, and VEGFD as well as the placental growth factor (PLGF) [13]. The most potent member is VEGFA [14], which acts as a key regulator of both physiological and pathological angiogenesis and modulates proliferation, median survival, and metastasis [15]. The human VEGFA gene is located on chromosome 6 , with multiple common SNPs in the promoter as well as the $5^{\prime}$ and 3 ' untranslated regions. It is organized into eight exons and seven introns [16]. Evidence of the association between VEGFA polymorphisms and susceptibility to cancer (including PTC [17]) has been shown in many previous investigations [18]. However, these sequence variants explain only a small fraction of the estimated heritability of diseases, and the replication of these findings can be difficult.

Due to the conflicting results on the role of VEGFA polymorphisms in thyroid diseases [17, 19], more studies are required to clarify these inconsistencies. Additionally, there is no report on the association between thyroid cancer and VEGFA polymorphisms in the Chinese population. In this study, we selected five tag SNPs in $V E G F A$ to investigate the association between thyroid cancer (PTC and $\mathrm{NG}$ ) and VEGFA polymorphisms in Northern Han Chinese.

\section{RESULTS}

Table 1 showed the age and gender distributions and proportions of the three groups of study participants. In total, 861 PTC patients (209 males and 652 females), 562 NG patients (138 males and 424 females) and 896 control subjects (220 males and 676 females) were enrolled. The median (P25-P75) age of PTC patients, NG patients and control subjects were 44(38-49), 49(42-56) and 43(38-49), respectively. The proportion of females was higher in both case groups, but this difference was not statistically significant $\left(\chi^{2}=0.023, P=0.989\right)$. We found statistically significant differences in the median age between cases and controls $(P<0.001)$. Multiple comparisons showed that subjects with $\mathrm{NG}$ were significantly older than other groups (both $P<0.001$ ). Genotype distributions of SNPs rs10434, rs25648 and rs3025040 in PTC and NG patients as well as controls fit the Hardy-Weinberg equilibrium (HWE). Genotype distributions of SNP rs3024997 were in HWE only in the PTC group. Genotype distributions of SNP rs833070 revealed deviation from $\mathrm{HWE}$ in all three groups. $10 \%$ of the samples were randomly selected to check polymorphism genotyping, and no genotyping errors were found.

The genotypic and allelic frequencies of five VEGFA polymorphisms are shown in Table 2. The $\mathrm{T}$ allele of SNP rs3025040 was less frequent in PTC $(17.58 \%)$ and NG (16.73\%) patients in comparison to control subjects $(20.03 \%)(P=0.050)$. With multinomial logistic regression analyses adjusted by age and sex, we found a significant association of SNP rs3025040 with NG [OR: 0.81(95\%CI:0.67-0.99); $P=0.042$ ]. No significant differences in allele frequencies of SNPs rs10434, rs25648, rs833070, and rs3024997 ( $P>0.05)$ were observed between PTC or NG patients and healthy controls. Genotype distributions of SNPs rs3024997 and rs3025040 appeared to be significantly different between cases and controls (both $P<0.05$ ).

We performed multinomial logistic regression analyses to determine the best inheritance model explaining the association between PTC or NG and the SNPs investigated, and the results are presented in Table 3. Our results revealed no significant association between PTC or NG and VEGFA SNPs rs10434, rs25648, and rs833070 $(P>0.05)$. In the TT vs. CC (PTC cases versus healthy controls) genotypic contrast for the SNP rs3025040, we found a significantly protective effect of the rs3025040 variant on PTC [OR: 0.57 (95\%CI: 0.33-0.98); $P=0.043]$. We also found a significantly protective effect of SNP rs3025040 on NG in the TC vs. CC genotypic contrast [OR:0.73 (95\%CI:0.57-0.93); $P=0.012]$. In the over dominant model (AG vs. AA+GG genotypes), we found a significant association between PTC risk and SNP rs3024997 [OR:1.21 (95\%CI:1.00-1.47); $P=0.045$ ] as well as between NG risk and SNP rs3024997 [OR:1.62 (95\%CI:1.30-2.02); $P<0.001]$. 
Table 2: Genotypic and allelic frequencies

\begin{tabular}{|c|c|c|c|c|c|c|}
\hline SNP & $\begin{array}{l}\text { Genotype/ } \\
\text { Allele }\end{array}$ & PTC(\%) & NG(\%) & control(\%) & $\chi^{2}$ & $P$ \\
\hline \multirow[t]{5}{*}{ rs 10434} & AA & $34(4.01)$ & $22(4.16)$ & $27(3.10)$ & 2.993 & 0.559 \\
\hline & GA & $285(33.65)$ & $171(32.33)$ & $271(31.15)$ & & \\
\hline & GG & $528(62.34)$ & $336(63.52)$ & $572(65.75)$ & & \\
\hline & A & $353(20.84)$ & $215(20.32)$ & $325(18.68)$ & 2.684 & 0.261 \\
\hline & G & 1341(79.16) & $843(79.68)$ & $1415(81.32)$ & & \\
\hline \multirow[t]{5}{*}{ rs 25648} & $\mathrm{CC}$ & $656(84.86)$ & 397(87.44) & $662(88.15)$ & - & $0.301 *$ \\
\hline & $\mathrm{CT}$ & $112(14.49)$ & $53(11.67)$ & $86(11.45)$ & & \\
\hline & $\mathrm{TT}$ & $5(0.65)$ & $4(0.88)$ & $3(0.40)$ & & \\
\hline & $\mathrm{C}$ & 1424(92.11) & $847(93.28)$ & 1410(93.87) & 3.733 & 0.152 \\
\hline & $\mathrm{T}$ & $122(7.89)$ & $61(6.72)$ & $92(6.13)$ & & \\
\hline \multirow[t]{5}{*}{ rs3024997 } & AA & $165(19.50)$ & $121(22.45)$ & $150(17.48)$ & 15.997 & 0.003 \\
\hline & AG & $418(49.41)$ & $234(43.41)$ & $466(54.31)$ & & \\
\hline & GG & 263(31.09) & $184(34.14)$ & $242(28.21)$ & & \\
\hline & A & $748(44.21)$ & $476(44.16)$ & $766(44.64)$ & 0.088 & 0.957 \\
\hline & G & $944(55.79)$ & $602(55.84)$ & $950(55.36)$ & & \\
\hline \multirow[t]{5}{*}{ rs3025040 } & $\mathrm{CC}$ & $574(67.29)$ & $394(70.48)$ & $571(64.09)$ & 10.741 & 0.030 \\
\hline & $\mathrm{TC}$ & $258(30.25)$ & $143(25.58)$ & $283(31.76)$ & & \\
\hline & $\mathrm{TT}$ & $21(2.46)$ & $22(3.94)$ & $37(4.15)$ & & \\
\hline & $\mathrm{C}$ & $1406(82.42)$ & $931(83.27)$ & $1425(79.97)$ & 6.010 & 0.050 \\
\hline & $\mathrm{T}$ & $300(17.58)$ & $187(16.73)$ & $357(20.03)$ & & \\
\hline \multirow[t]{5}{*}{ rs 833070} & AA & $204(24.14)$ & $121(22.79)$ & $228(25.97)$ & 3.693 & 0.449 \\
\hline & $\mathrm{AG}$ & $146(17.28)$ & $108(20.34)$ & $156(17.77)$ & & \\
\hline & GG & $495(58.58)$ & $302(56.87)$ & $494(56.26)$ & & \\
\hline & A & $554(32.78)$ & $350(32.96)$ & $612(34.85)$ & 1.936 & 0.380 \\
\hline & G & $1136(67.22)$ & $712(67.04)$ & $1144(65.15)$ & & \\
\hline
\end{tabular}

*Fisher's Exact Test; Bold numbers mean a significant association.

To assess the combined effects of SNPs in VEGFA on PTC and NG, we performed haplotype analyses and presented the results in Table 4 and Supplementary Tables 1-3. Our results revealed that a VEGFA haplotype, consisting of the minor alleles of SNPs rs3024997 and rs3025040 as well as the major alleles of SNPs rs 10434, rs25648, and rs833070, occurred less frequent in PTC and NG cases than in controls $(5.91 \%, 5.26 \%$ vs. $7.33 \%$, respectively). However, these differences were not statistically significant [PTC vs. Control: OR:0.73 (95\%CI: 0.53-1.00), $P=0.053$; NG vs. Control: OR: $0.66(95 \%$ CI: $0.44-1.00), P=0.050]$. Haplotype AT (consisting of alleles of SNPs rs3024997 and rs3025040) showed a protective effect on NG $[8.47 \%$ (NG) $v s .12 .00 \%$
(Control): $P=0.009]$. Haplotype TG (consisting of allele of SNPs rs25648 and rs833070) played a risk role in PTC [3.97\% (PTC) vs. 2.64\% (controls): $P=0.049$ ). Haplotype GTT (consisting of alleles of SNPs rs833070, rs3025040 and rs3024997) could predict the risk of progression to NG $(P=0.025$ ), while haplotype GTA (consisting of alleles of SNPs rs10434, rs3025040 and rs3024997) predicted the protection of progression to NG $(P=0.011)$. Haplotypes GGAT and AGAC (consisting alleles of SNPs rs833070, rs10434, rs3024997, and rs3025040) conferred protection to both NG $(P=0.044)$ and PTC ( $P=0.027)$, but haplotype GGGT (rs833070, rs10434, rs3024997, rs3025040) conferred risk for NG $(P=0.020)$. Other haplotypes did not show significant associations with PTC or NG. 
Table 3: Univariable multinomial Logistic Regression analysis of the association between the $V E G F A$ polymorphism genotypes and alleles with PTC and NG

\begin{tabular}{|c|c|c|c|c|c|c|}
\hline \multirow{2}{*}{ SNP } & \multirow{2}{*}{ Genotype } & \multirow{2}{*}{$\begin{array}{c}\text { Inheritance } \\
\text { model }\end{array}$} & \multicolumn{2}{|c|}{ PTC vs. control } & \multicolumn{2}{|c|}{ NG vs. control } \\
\hline & & & OR(95\%CI) & $P$ & OR(95\% CI) & $P$ \\
\hline \multirow[t]{2}{*}{ rs 10434} & GG & Dominant & 1.00 (ref) & & 1.00 (ref) & \\
\hline & $\mathrm{AA}+\mathrm{GA}$ & & $1.15(0.94-1.40)$ & 0.168 & $1.08(0.86-1.36$ & 0.517 \\
\hline \multirow[t]{2}{*}{ rs 25648} & $\mathrm{CC}$ & Dominant & 1.00 (ref) & & 1.00 (ref) & \\
\hline & $\mathrm{CT}+\mathrm{TT}$ & & $1.33(0.98-1.78)$ & 0.063 & $1.08(0.75-1.55)$ & 0.688 \\
\hline \multirow[t]{2}{*}{ rs3024997 } & $\mathrm{AA}+\mathrm{GG}$ & Over dominant & 1.00 (ref) & & 1.00 (ref) & \\
\hline & $\mathrm{AG}$ & & $1.21(1.00-1.47)$ & 0.045 & $1.62(1.30-2.02)$ & $<\mathbf{0 . 0 0 1}$ \\
\hline \multirow[t]{3}{*}{ rs3025040 } & $\mathrm{CC}$ & Codominant & 1.00 (ref) & & 1.00 (ref) & \\
\hline & $\mathrm{TC}$ & & $0.91(0.74-1.12)$ & 0.378 & $0.73(0.57-0.93)$ & 0.012 \\
\hline & $\mathrm{TT}$ & & $0.57(0.33-0.98)$ & 0.043 & $0.94(0.54-1.64)$ & 0.815 \\
\hline \multirow[t]{2}{*}{ rs833070 } & $\mathrm{AA}+\mathrm{GG}$ & Over dominant & 1.00 (ref) & & 1.00 (ref) & \\
\hline & $\mathrm{AG}$ & & $0.96(0.75-1.24)$ & 0.772 & $1.24(0.94-1.64)$ & 0.134 \\
\hline
\end{tabular}

Odds ratio (OR) 95\% CI and $P$ values was adjusted for age and gender; Bold numbers mean a significant association.

Table 4: Haplotyping analysis of $V E G F A$ SNPs in PTC and NG

\begin{tabular}{|c|c|c|c|c|c|c|c|c|c|c|c|c|}
\hline \multirow[t]{2}{*}{ Block } & \multicolumn{5}{|c|}{ Haplotype* } & \multicolumn{3}{|c|}{ Frequency $(\%)$} & \multicolumn{2}{|c|}{ PTC vs. control } & \multicolumn{2}{|c|}{ NG vs. control } \\
\hline & 1 & 2 & 3 & 4 & 5 & PTC & NG & control & OR(95\%CI) & $P$ & OR(95\% CI) & $P$ \\
\hline 1 & G & $\mathrm{C}$ & A & $\mathrm{C}$ & G & 24.33 & 24.19 & 21.98 & 1.00 (ref) & & 1.00 (ref) & \\
\hline 2 & G & $\mathrm{C}$ & G & $\mathrm{C}$ & G & 17.41 & 16.54 & 17.82 & $0.89(0.70-1.12)$ & 0.310 & $0.89(0.67-1.18)$ & 0.410 \\
\hline 3 & $\mathrm{~A}$ & $\mathrm{C}$ & G & $\mathrm{C}$ & G & 11.56 & 12.66 & 12.5 & $0.83(0.64-1.08)$ & 0.175 & $0.94(0.69-1.28)$ & 0.676 \\
\hline 4 & G & $\mathrm{C}$ & G & $\mathrm{C}$ & A & 10.28 & 10.9 & 9.84 & $0.94(0.71-1.24)$ & 0.660 & $1.04(0.75-1.45)$ & 0.816 \\
\hline 5 & G & $\mathrm{C}$ & A & $\mathrm{T}$ & G & 5.91 & 5.26 & 7.33 & $0.73(0.53-1.00)$ & 0.053 & $0.66(0.44-1.00)$ & 0.050 \\
\hline 6 & G & $\mathrm{C}$ & A & $\mathrm{C}$ & A & 5.38 & 6.14 & 7.33 & $0.67(0.48-0.93)$ & 0.016 & $0.77(0.52-1.14)$ & 0.193 \\
\hline 7 & G & $\mathrm{C}$ & G & $\mathrm{T}$ & A & 4.44 & 4.26 & 4.31 & $0.93(0.63-1.36)$ & 0.700 & $1.01(0.63-1.61)$ & 0.976 \\
\hline 8 & G & $\mathrm{C}$ & A & $\mathrm{T}$ & A & 4.03 & 3.63 & 4.74 & $0.80(0.54-1.17)$ & 0.242 & $0.67(0.41-1.08)$ & 0.098 \\
\hline 9 & A & $\mathrm{C}$ & A & $\mathrm{C}$ & G & 4.3 & 4.76 & 3.66 & $1.06(0.71-1.59)$ & 0.758 & $1.17(0.73-1.88)$ & 0.503 \\
\hline 10 & A & $\mathrm{C}$ & G & $\mathrm{C}$ & A & 2.49 & 1.63 & 2.08 & $1.12(0.67-1.87)$ & 0.656 & $0.71(0.36-1.42)$ & 0.333 \\
\hline 11 & G & $\mathrm{T}$ & G & $\mathrm{C}$ & A & 2.02 & 1.75 & 2.51 & $0.73(0.44-1.23)$ & 0.237 & $0.65(0.33-1.25)$ & 0.192 \\
\hline 12 & G & $\mathrm{C}$ & G & $\mathrm{T}$ & G & 1.61 & 2.01 & 1.65 & $0.89(0.49-1.61)$ & 0.698 & $1.16(0.59-2.28)$ & 0.681 \\
\hline 13 & G & $\mathrm{T}$ & G & $\mathrm{C}$ & G & 1.61 & 1.88 & 1.29 & $1.12(0.60-2.11)$ & 0.724 & $1.35(0.65-2.79)$ & 0.420 \\
\hline 14 & A & $\mathrm{T}$ & G & $\mathrm{C}$ & A & 1.61 & 1.13 & 0.65 & $2.15(0.98-4.72)$ & 0.058 & $1.79(0.69-4.69)$ & 0.234 \\
\hline 15 & A & $\mathrm{T}$ & G & $\mathrm{C}$ & G & 1.28 & 1.13 & 0.93 & $1.29(0.63-2.67)$ & 0.484 & $1.08(0.45-2.61)$ & 0.863 \\
\hline Rare $^{\#}$ & - & - & - & - & - & 1.75 & 2.13 & 1.36 & $1.17(0.63-2.15)$ & 0.623 & $1.44(0.72-2.88)$ & 0.306 \\
\hline
\end{tabular}

Odds ratio (OR) 95\% CI and $P$ values was adjusted for age and gender.

* SNPs are as follows: 1, rs10434; 2, rs25648; 3, rs3024997; 4, rs3025040; 5, rs833070.

\# haplotypes with frequencies $<0.01$. 


\section{DISCUSSION}

By performing the present study, we demonstrated the relationship between five SNPs of the VEGFA gene with PTC and NG in a Han Chinese population. In our study, $76 \%$ of patients with PTC were female. The median age of 44 years as revealed in this present study is consistent with the findings reported by Shi et al. in 2012 [20]. We found that SNPs rs3024997 and rs3025040 were associated with PTC or NG susceptibility. No association between PTC or NG and other SNPs (rs10434, rs25648, and rs833070) was observed. Haplotype analyses suggested a combined effect of SNPs in VEGFA on the risk of PTC or NG.

The basis of some thyroid disorders, including PTC and NG, may reflect the effects of polymorphisms in multiple genes. PTC has relationships with several genes including FOXE1 [21], BRAF V600E [22], and Fas [23]. SNPs in VEGFA are involved in several types of cancers, such as the bladder [24], esophageal [25], thyroid [11] and gastric [26] cancers. VEGF influences cell angiogenesis, proliferation, and migration, and plays an important role in thyroid cancer cell growth and distant metastasis [27]. The molecular pathogenesis of thyroid cancer involves genetic alterations and their relationship with signaling pathways, including the mitogen activated protein kinase (MAPK) [28]. The MAPK signal pathway is involved in the regulation of cell proliferation and differentiation [29]. Activation of the MAPK pathway mainly drives the development of PTC [30]. The binding VEGFA to its receptor can activate the MAPK pathway [31].

A number of studies have evaluated that age and female gender are risk factors for thyroid cancer [32, 33]. The relationship between VEGFA polymorphisms and thyroid diseases had been analyzed in many previous studies. Vural et al. investigated the association between VEGFA polymorphisms rs699947, rs833061 and rs2010963 and susceptibility to Graves' disease (GD) risk, and reported that VEGFA rs2010963 was a risk factor for GD, and SNP rs699947 C allele was associaetd with elevated autoantibody levels [34]. Marotta et al. examined four SNPs (rs699947, rs833061, rs2010963, rs3025039) in VEGFA and demonstrated that the minor homozygous genotypes AA of SNPs rs699947 and CC of SNP rs833061 $\mathrm{CC}$ exerted a protective effect on differentiated thyroid cancer (DTC), and haplotype ACG (consisting of alleles of VEGFA SNPs rs699947, rs833061, and rs2010963) was protective to DTC [35]. Niccolai et al. reported that SNP rs3025039 (+936C $>$ T) of VEGFA was not associated with NG in patients from an area with a mild iodine deficiency [36]. Moreover, Salajegheh et al. revealed that SNPs rs3025039 and rs2010963 $(+405 \mathrm{C}>\mathrm{G})$ of VEGFA might predict the malignancy progresses of thyroid tumor [17]. The study by Hsiao et al [19] suggested that VEGFA rs699947 increased the risk of thyroid cancer and lymph node metastasis in males, but not in females. However, these researchers found no significant associations between thyroid cancer risk and VEGFA SNPs rs3025039 and rs2010963. Our results showed that individuals carrying SNP rs3025040-T allele had a 0.81-fold decrease risk of developing to NG. The heterozygote AG of SNP rs3024997 was associated with the risk of developing PTC and NG compared with healthy controls. Our study revealed that the TT genotype of SNP rs3025040 protected against the risk of PTC, and the TC genotype of this same variant was protective against NG risk in our study population. There are several explanations for our findings. Sufficient evidence indicates that VEGFA plays a complicated and critical role in thyroid disorder [37], and that VEGFA polymorphisms can contribute to the interindividual variants in VEGF expression [38]. The inconsistent reports on the role of VEGFA in thyroid disorders might be due to the effects of the different polymorphisms in this gene. We had $25.01 \%$ to $92.15 \%$ power to detect genotype distribution differences between case and control groups. The inadequate power may be the cause of negative results in genetic association studies. Since all patients were recruited in hospital, some potential interfering factors, such as Berkson bias, were not controlled, which might induce bias.

\section{MATERIALS AND METHODS}

\section{Study participants}

We recruited study participants from the ChinaJapan Union Hospital, Jilin University, Changchun, from August 2012 to December 2014. All cases were unrelated Northern Han Chinese who made clinic visits at the recruitment center. Patients were confirmed by pathologic examinations based on the American Thyroid Association (ATA) guidelines [39]. We excluded specimens confirmed with nodular hyperplasia, anaplastic carcinoma, follicular carcinoma, and follicular variant of PTC. Controls were selected from the general population. They were free from thyroid diseases, cancers, or any other endocrine system diseases. The study included 2,319 participants, comprising 1,423 cases (861 PTC patients and $562 \mathrm{NG}$ patients) and 896 controls.

This study adhered to the tenets of the Declaration of Helsinki. All study subjects made written informed consents before entering the study. Ethics approval was obtained from the Human Research Ethics Committee of School of Public Health, Jilin University.

\section{SNP selection}

VEGFA SNPs for the Han Chinese population were chosen from the online HapMap database (HapMap Data Rel 24/phaseII Nov08, on NCBI B36 Assembly, dbSNP b126). Tag SNPs were obtained by implementing the software Haploview version 4.2 (Broad Institute, 
Cambridge, MA). For all SNPs investigated, we set the threshold for minor allele frequency (MAF) at 0.1, and correlation coefficiency $\left(\mathrm{r}^{2}\right)$ more than 0.8. Five tag SNPs in the VEGFA gene were selected, and among them, SNPs rs 10434 and rs3025040 were located in the 3' untranslated region (3'UTR), SNP rs25648 was located in the exon, and the others (rs3024997and rs833070) were located in the introns.

\section{Genomic DNA extraction}

Genomic DNA was extracted from peripheral blood using the DNA extraction kit (Beijing Kangwei Century Biotech Co., Ltd., China). The concentration and purity of DNA samples were determined by spectrophotometer (Shimadzu, Kyoto, Japan).

\section{SNP genotyping}

SNP genotyping was performed using the Sequenom MassARRAY platform (San Diego, CA, USA). Genomic DNA amplification was conducted using specific forward and reverse primer pairs as follows: For rs3024997, the primers were sense 5'-ACGTTGGATGCTCTGTAATG CCACTCTTTG-3' and antisense 5'-ACGTTGGATGTC AAACACAGTAGGAGGGAC -3'. For rs3025040, the primers were sense 5'-ACGTTGGATGAGATCACAG GTACAGGGATG-3' and antisense 5'-ACGTTGGATG ATCCCCAAAGCACAGCAATG-3'. For rs833070, the primers were sense 5'-ACGTTGGATGTCAGCCTAATG GGATCTCTC-3' and antisense 5'-ACGTTGGATGAGT TCACAGCACCCGAACAT-3'. For rs25648, the primers were sense 5'-ACGTTGGATGGCACCCAAGACAGCA GAAAG-3' and antisense 5'-ACGTTGGATGCACAGC CCGAGCCGGAGAG-3'. For rs 10434, the primers were sense 5'-ACGTTGGATGTCTCACCTGCTTCTGAGT TG-3' and antisense 5'-ACGTTGGATGGGCTGCTTC TTCCAACAATG-3'. PCR comprised an initial cycle at $94^{\circ} \mathrm{C}$ for 15 minutes to perform a hot-start, then 45 cycles at $94^{\circ} \mathrm{C}$ for 20 seconds, $56^{\circ} \mathrm{C}$ for 30 seconds, $72^{\circ} \mathrm{C}$ for 60 seconds, and 1 cycle at $72^{\circ} \mathrm{C}$ for 3 minutes in the final reaction. Then, the shrimp alkaline phosphatase (SAP) reaction was performed by incubating the PCR product with SAP (Sequenom, Inc., San Diego, CA, USA) at $37^{\circ} \mathrm{C}$ for 40 minutes, followed by inactivation at $85^{\circ} \mathrm{C}$ for five minutes. The iPLEX extension reaction was performed at $94^{\circ} \mathrm{C}$ for 30 seconds and $94^{\circ} \mathrm{C}$ for five seconds, followed by 40 cycles at $52^{\circ} \mathrm{C}$ for five seconds and 5 cycles at $80^{\circ} \mathrm{C}$ for five seconds and at $72^{\circ} \mathrm{C}$ for three minutes. The PCR products were desalted by the addition of resin in a 384-dimple plate, mixed, resuspended, and centrifuged to separate the extension products from the resin. The completed products were analyzed using the MassARRAY Typer software version 4.0 (Sequenom, USA). Due to the poor quality of some biological samples available for DNA extraction, the genotyping successful rate was not $100 \%$ for each SNP.

\section{Statistical analyses}

Conformation with HWE in each group was examined by the Chi-square test. The proportions of genotypes and allelic frequencies were analyzed. Continuous variables (non-normal distribution) were presented as median and percentile values (P25-P75). We analyzed categorical variables using Chi-square or Fisher's exact tests as applicable. Multinomial logistic regression analysis was employed to obtain odds ratios (ORs), 95\% confidence intervals (CIs), and $P$-values. Age and sex were adjusted as covariates in the statistical analyses. Haplotypes comprising alleles of the five SNPs were evaluated, and each haplotype frequency was inferred to identify differences between groups. The statistical significance level was set at 0.05 , and all statistical tests were two sided. We performed all statistical analyses using R software (version 3.24) (R Foundation for Statistical Computing, Vienna, Austria).

\section{CONCLUSION}

In conclusion, we investigated the association of tag SNPs in VEGFA (which is involved in the MAPK pathway) and PTC and NG in a Han Chinese population. We found that VEGFA polymorphisms might play an important role in PTC and NG. Carriers of rs3025040-T allele had a decreased risk of developing NG. SNPs rs3024997 and rs3025040 in VEGFA were significantly associated with PTC and NG. Combined effect of SNPs in VEGFA might influence the risk of PTC and NG. Since the exact mechanism or roles of VEGFA SNPs on thyroid disease are still unknown, further studies with larger samples are needed to validate our findings. In addition, sex-specific effects of VEGFA SNPs, ethnic differences and gene-environment interaction in the association between VEGFA single nucleotide variants and thyroid cancer merit further investigation.

\section{ACKNOWLEDGMENTS}

This study was supported by an NSFC grant (No. 81673253), the Norman Bethune Program of Jilin University (2015227) and the young Program of Jilin Provincial Health Department (2014Q027).

\section{CONFLICTS OF INTEREST}

The authors declare that no conflicts of interests exists.

\section{REFERENCES}

1. Ito Y, Miyauchi A, Kobayashi K, Miya A. Prognosis and growth activity depend on patient age in clinical and 
subclinical papillary thyroid carcinoma. Endocrine journal. 2014; 61:205-213.

2. Davies L, Welch H. Current thyroid cancer trends in the united states. JAMA Otolaryngology-Head \& Neck Surgery. 2014; 140:317-322.

3. Chen P, Sun R, Pu Y, Bai P, Yuan F, Liang Y, Zhou B, Wang Y, Sun Y, Zhu J, Zhang L, Gao L. Pri-Mir-34b/C and Tp-53 Polymorphisms are Associated With The Susceptibility of Papillary Thyroid Carcinoma: A Case-Control Study. Medicine (Baltimore). 2015; 94:e1536.

4. Fallah M, Pukkala E, Tryggvadottir L, Olsen JH, Tretli S, Sundquist K, Hemminki K. Risk of thyroid cancer in firstdegree relatives of patients with non-medullary thyroid cancer by histology type and age at diagnosis: a joint study from five Nordic countries. Journal of medical genetics. 2013; 50:373-382.

5. Handkiewicz-Junak D, Swierniak M, Rusinek D, OczkoWojciechowska M, Dom G, Maenhaut C, Unger K, Detours V, Bogdanova T, Thomas G, Likhtarov I, Jaksik R, Kowalska M, Chmielik E, Jarzab M, Swierniak A, et al. Gene signature of the post-Chernobyl papillary thyroid cancer. Eur J Nucl Med Mol Imaging. 2016; 43:1267-1277.

6. Kim SK, Kim DK, Oh IH, Song JY, Kwon KH, Choe BK, Kim YH. A missense polymorphism (rs11895564, Ala380Thr) of integrin alpha 6 is associated with the development and progression of papillary thyroid carcinoma in Korean population. Journal of the Korean Surgical Society. 2011; 81:308-315.

7. Cummings AL, Goldfarb M. Thyroid carcinoma metastases to axillary lymph nodes: report of two rare cases of papillary and medullary thyroid carcinoma and literature review. Endocrine practice. 2014; 20:e34-e37.

8. Baloch ZW, LiVolsi VA. Current role and value of fineneedle aspiration in nodular goitre. Best practice \& research Clinical endocrinology \& metabolism. 2014; 28:531-544.

9. Syrenicz A, Koziolek M, Ciechanowicz A, Sieradzka A, Binczak-Kuleta A, Parczewski M. New insights into the diagnosis of nodular goiter. Thyroid research. 2014; 7:6.

10. Derwahl M, Studer H. Nodular goiter and goiter nodules: Where iodine deficiency falls short of explaining the facts. Experimental and clinical endocrinology \& diabetes. 2001; 109:250-260.

11. Cho SH, Kim SK, Kwon E, Park HJ, Kwon KH, Chung JH. Polymorphism of IGF1R is associated with papillary thyroid carcinoma in a Korean population. Journal of interferon \& cytokine research. 2012; 32:401-406.

12. Kang J, Deng XZ, Fan YB, Wu B. Relationships of FOXE1 and ATM genetic polymorphisms with papillary thyroid carcinoma risk: a meta-analysis. Tumour biology. 2014; 35:7085-7096.

13. Claesson-Welsh L, Welsh M. VEGFA and tumour angiogenesis. Journal of internal medicine. 2013; 273:114-127.
14. Rapisarda A, Melillo G. Role of the VEGF/VEGFR axis in cancer biology and therapy. Advances in cancer research. 2012; 114:237-267.

15. Andreozzi M, Quagliata L, Gsponer JR, Ruiz C, Vuaroqueaux V, Eppenberger-Castori S, Tornillo L, Terracciano LM. VEGFA gene locus analysis across 80 human tumour types reveals gene amplification in several neoplastic entities. Angiogenesis. 2014; 17:519-527.

16. Hansen TF, Jakobsen A. Clinical implications of genetic variations in the VEGF system in relation to colorectal cancer. Pharmacogenomics. 2011; 12:1681-1693.

17. Salajegheh A, Smith RA, Kasem K, Gopalan V, Nassiri MR, William R, Lam AK. Single nucleotide polymorphisms and mRNA expression of VEGF-A in papillary thyroid carcinoma: potential markers for aggressive phenotypes. European journal of surgical oncology. 2011; 37:93-99.

18. Langsenlehner U, Hofmann G, Renner W, Gerger A, Krenn-Pilko S, Thurner EM, Krippl P, Langsenlehner T. Association of vascular endothelial growth factor--a gene polymorphisms and haplotypes with breast cancer metastases. Acta oncologica (Stockholm, Sweden). 2015; 54:368-376.

19. Hsiao PJ, Lu MY, Chiang FY, Shin SJ, Tai YD, Juo SH. Vascular endothelial growth factor gene polymorphisms in thyroid cancer. The Journal of endocrinology. 2007; 195:265-270.

20. Shi L, Li Y, Guan H, Li C, Shi L, Shan Z, Teng W. Usefulness of serum thyrotropin for risk prediction of differentiated thyroid cancers does not apply to microcarcinomas: results of 1,870 Chinese patients with thyroid nodules. Endocrine journal. 2012; 59:973-980.

21. Landa I, Ruiz-Llorente S, Montero-Conde C, Inglada-Perez L, Schiavi F, Leskela S, Pita G, Milne R, Maravall J, Ramos I, Andia V, Rodriguez-Poyo P, Jara-Albarran A, Meoro A, del Peso C, Arribas L, et al. The variant rs1867277 in FOXE1 gene confers thyroid cancer susceptibility through the recruitment of USF1/USF2 transcription factors. PLoS genetics. 2009; 5:e1000637.

22. Xing M. BRAF mutation in papillary thyroid cancer: pathogenic role, molecular bases, and clinical implications. Endocrine reviews. 2007; 28:742-762.

23. Basolo F, Giannini R, Faviana P, Fontanini G, Patricelli Malizia A, Ugolini C, Elisei R, Miccoli P, Toniolo A. Thyroid papillary carcinoma: preliminary evidence for a germ-line single nucleotide polymorphism in the Fas gene. The Journal of endocrinology. 2004; 182:479-484.

24. Garcia-Closas M, Malats N, Real FX, Yeager M, Welch R, Silverman D, Kogevinas M, Dosemeci M, Figueroa J, Chatterjee N, Tardon A, Serra C, Carrato A, GarciaClosas R, Murta-Nascimento C, Rothman N, et al. Largescale evaluation of candidate genes identifies associations between VEGF polymorphisms and bladder cancer risk. PLoS genetics. 2007; 3:e29. 
25. Bradbury PA, Zhai R, Ma C, Xu W, Hopkins J, Kulke MJ, Asomaning K, Wang Z, Su L, Heist RS, Lynch TJ, Wain JC, Christiani D, Liu G. Vascular endothelial growth factor polymorphisms and esophageal cancer prognosis. Clinical cancer research. 2009; 15:4680-4685.

26. Guan X, Zhao H, Niu J, Tang D, Ajani JA, Wei Q. The VEGF $-634 \mathrm{G}>\mathrm{C}$ promoter polymorphism is associated with risk of gastric cancer. BMC gastroenterology. 2009; 9:77.

27. Lin JD, Chao TC. Vascular endothelial growth factor in thyroid cancers. Cancer Biotherapy \& Radiopharmaceuticals. 2005; 20:648-661.

28. Hou P, Liu D, Shan Y, Hu S, Studeman K, Condouris S, Wang Y, Trink A, El-Naggar AK, Tallini G, Vasko V, Xing M. Genetic alterations and their relationship in the phosphatidylinositol 3-kinase/Akt pathway in thyroid cancer. Clinical cancer research. 2007; 13:1161-1170.

29. Zhang Q, Song F, Zheng H, Zhu X, Song F, Yao X, Zhang L, Chen K. Association between single-nucleotide polymorphisms of BRAF and papillary thyroid carcinoma in a Chinese population. Thyroid. 2013; 23:38-44.

30. Xing M. Molecular pathogenesis and mechanisms of thyroid cancer. Nature reviews Cancer. 2013; 13: 184-199.

31. Vlajnic T, Andreozzi MC, Schneider S, Tornillo L, Karamitopoulou E, Lugli A, Ruiz C, Zlobec I, Terracciano L. VEGFA gene locus (6p12) amplification identifies a small but highly aggressive subgroup of colorectal cancer [corrected] patients. Modern pathology. 2011; 24:1404-1412.

32. Su X, Li Z, He C, Chen W, Fu X, Yang A. Radiation exposure, young age, and female gender are associated with high prevalence of RET/PTC1 and RET/PTC3 in papillary thyroid cancer: a meta-analysis. Oncotarget. 2016; 7:1671616730. doi: 10.18632/oncotarget. 7574 .

33. Alhozali A, Al-Ghamdi A, Alahmadi J. Pattern of Thyroid Cancer at King Abdulaziz University Hospital, Jeddah: A 10-Year Retrospective Study. Open Journal of Endocrine and Metabolic Diseases. 2016; 6:121-125.
34. Vural P, Baki M, Dogru-Abbasoglu S, Ozderya A, Karadag B, Uysal M. Vascular endothelial growth factor polymorphisms increase the risk of developing Graves' disease. International immunopharmacology. 2012; 14:133-137.

35. Marotta V, Sciammarella C, Capasso M, Testori A, Pivonello C, Chiofalo MG, Gambardella C, Grasso M, Antonino A, Annunziata A, Macchia PE, Pivonello R, Santini L, Botti G, Losito S, Pezzullo L, et al. Germline polymorphisms of the VEGF-pathway predict recurrence in non-advanced differentiated thyroid cancer. The Journal of clinical endocrinology and metabolism (2016). doi: 10.1210/jc.2016-2555.

36. Niccolai F, Molinaro A, De Marco G, Agretti P, Di Cosmo C, Piaggi P, Pinchera A, Vitti P, Bocci G, Tonacchera M. Frequency of polymorphisms in the VEGF, VEGFR and HIF genes in normal subjects and patients with nodular goiter from an area with mild iodine deficiency. European Thyroid Journal. 2012; 1:157.

37. Celano M, Sponziello M, Tallini G, Maggisano V, Bruno R, Dima M, Di Oto E, Redler A, Durante C, Sacco R, Filetti $\mathrm{S}$, Russo D. Increased expression of pro-angiogenic factors and vascularization in thyroid hyperfunctioning adenomas with and without TSH receptor activating mutations. Endocrine. 2013; 43:147-153.

38. Kim DH, Xu W, Kamel-Reid S, Liu X, Jung CW, Kim $\mathrm{S}$, Lipton JH. Clinical relevance of vascular endothelial growth factor (VEGFA) and VEGF receptor (VEGFR2) gene polymorphism on the treatment outcome following imatinib therapy. Annals of oncology. 2010; 21:1179-1188.

39. Cooper DS, Doherty GM, Haugen BR, Kloos RT, Lee SL, Mandel SJ, Mazzaferri EL, McIver B, Pacini F, Schlumberger M, Sherman SI, Steward DL, Tuttle RM. Revised American Thyroid Association management guidelines for patients with thyroid nodules and differentiated thyroid cancer. Thyroid. 2009; 19:1167-1214. 